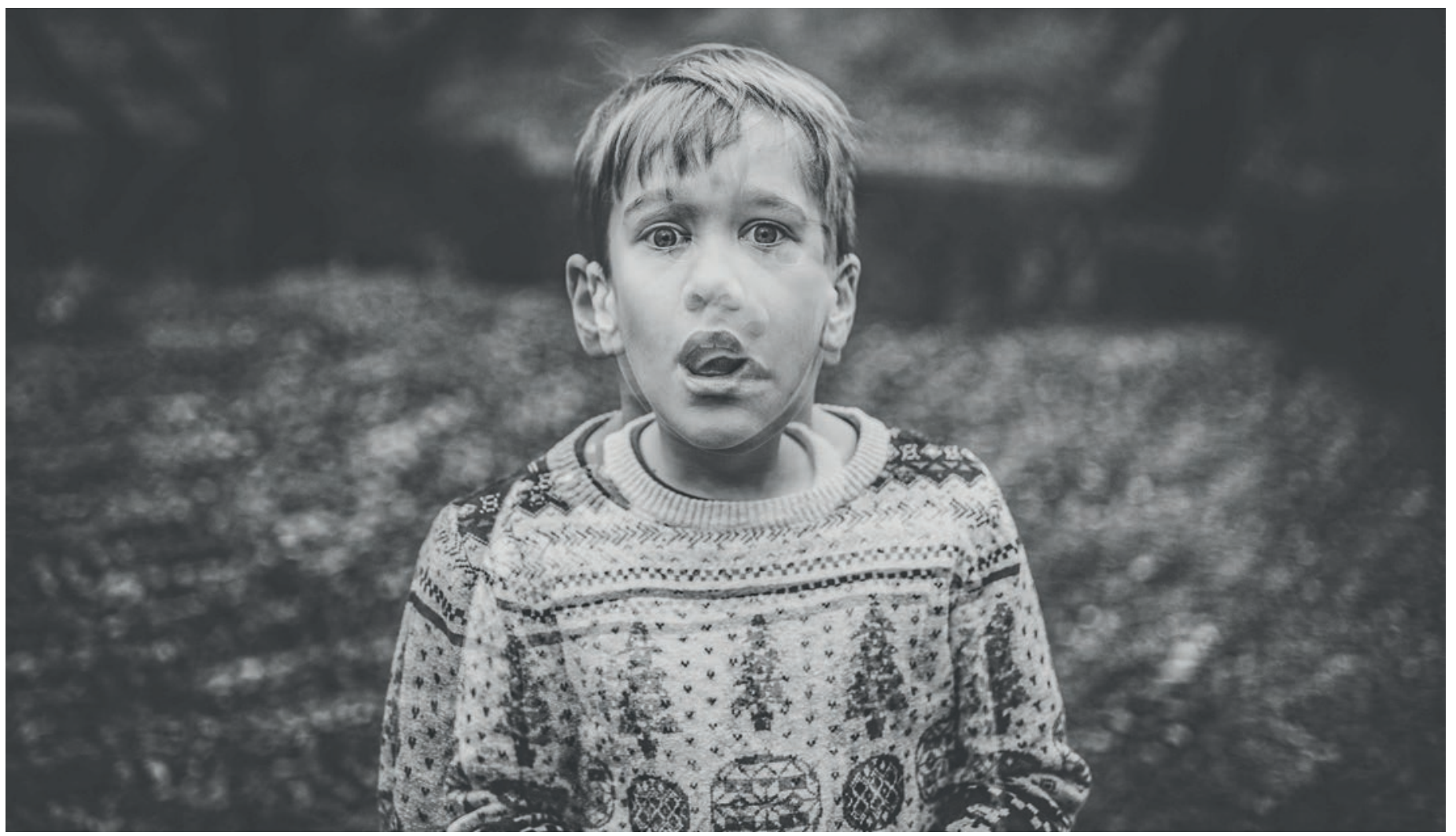

\title{
Chefvisite aus der Horizontalen
}

\section{Hans-Peter Mösch}

Dr. med., Facharzt für Allgemeine Innere Medizin, Wila

Wie fühlt es sich an, aus dem Koma aufzuwachen, nur Stimmen zu hören und nicht zu wissen, wo man ist und was los ist? Der Arzt Hans-Peter Mösch berichtet von seiner ersten - und schlimmsten - Chefvisite, die er 1970 als junger Patient erlebte.

Woher kam plötzlich dieses helle Licht? Eben war doch noch alles dunkel gewesen. Oder nein, ich wusste eigentlich gar nicht, wie es vorher gewesen war. Krampfhaft versuchte ich, mich zu erinnern. Ich war doch auf dem Weg zur Schule, ja heute hatten wir eine Lateinprüfung.

Aber ich sass ja nicht auf meinem Mofa, wo war ich? Wieder dieses helle Licht, nun hörte ich auch Stimmen, leise und gedämpft. Eine Frauenstimme sagte etwas von Pupille. Ich versuchte, mich zu konzentrieren, aber das war so schwierig.

Ich fühlte mich, als ob ich aus dem tiefen Wasser an die Oberfläche schwimmen müsste. Dabei merkte ich aber, dass ich mich gar nicht bewegen konnte. Wieder hörte ich eine Stimme, diesmal war es eine kräftige Männerstimme: «Er atmet gegen die Maschine.» Dann wieder die leise Frauenstimme, irgendetwas von Spritzen. Würde ich jetzt eine Spritze bekommen? Plötzlich versank ich erneut in der Tiefe, alles wurde schwarz.

\section{Verworrene Stimmen}

Wieder versuchte ich krampfhaft zu erwachen, denn es war so eigenartig hell um mich herum. Ich hörte Stimmen, versuchte, die Augen zu öffnen, aber es gelang mir nicht. Ich versuchte zu sprechen, dann zu schreien, aber es ging nicht. Ich hatte Angst. Irgendjemand machte etwas an mir, ich spürte ein Kneifen im Fuss, versuchte wieder die Augen zu öffnen, mich zu bewegen, aber alle Anstrengung war umsonst.

Da war sie wieder, die kräftige Männerstimme: «Immer noch im Koma", sagte sie, "schon die vierte Woche». Eine andere Männerstimme: «Die Halswirbelsäule müsste operiert werden, sie ist instabil», dann wieder die erste Stimme: «Das bringt nichts, keine Chance.» Dann eine zitternde Frauenstimme, sie kam mir sehr bekannt vor - konnte das die Stimme meiner Mutter sein? Sie fragte: «Kann er denn wieder gesund werden?» Daraufhin, fast unangenehm laut, die erste 
Stimme: "Seien Sie froh, wenn er stirbt, mit dieser schweren Hirnverletzung wird er nie mehr normal sein.» Ein leises Weinen und wieder die erste Stimme: «Warten wir einfach ab, vielleicht löst sich das Problem von selbst. Der Diadem-Gips bleibt, auch die Gurten, damit sich der Junge nicht bewegen kann, falls er doch aus dem Koma erwacht, wegen der Halswirbelsäule.» "Gerade bei jungen Menschen habe ich schon erstaunliche Rehabilitationen gesehen, ich denke, man sollte die Halswirbelsäule so schnell wie möglich operieren", sagte eine andere Männerstimme mit italienischem Akzent. Keine Antwort auf diese Bemerkung. Dann hörte ich Schritte, mehrere Stimmen, verstand sie aber nicht, sie wurden immer leiser, dann ein Geräusch, als ob eine Tür ins Schloss gezogen würde.

Hatten sie von mir gesprochen? Sollte ich sterben? Das

«Seien Sie froh, wenn er stirbt, mit dieser schweren Hirnverletzung wird er nie mehr normal sein.»

war doch nicht möglich, ich war doch auf dem Schulweg, wollte heute meine Lateinprüfung schreiben. Aber ich kam nicht weg von diesen Worten: Sterben, nicht mehr normal sein, keine Chance. Sie hatten sich bei mir eingebrannt, obwohl ich ständig damit kämpfte, wach zu bleiben. Ich war erst 14 Jahre alt, sollte mein Leben schon zu Ende sein? Ich hatte noch gar nicht richtig gelebt. Alles in mir sträubte sich, dem zu glauben, was ich soeben gehört hatte. Ich war voller Verzweiflung, Angst, und ich fühlte mich ganz allein damit. Erneut versuchte ich zu schreien, aber es ging nicht. Da versank ich plötzlich wieder im Nebel, aus dem ich versucht hatte, aufzutauchen.

\section{Das erste Mal die Augen öffnen}

Ein Rütteln weckte mich. Wo war ich? Ach ja, auf dem Schulweg. Mit dem Mofa musste es ja rütteln. Aber alles war so dunkel. Die Lateinprüfung, erinnerte ich mich. Ich versuchte, die Vokabeln im Kopf zu repetieren, aber es kam mir kein Wort mehr in den Sinn aus der Lektion, die ich gestern gelernt hatte. Nur ein Satz aus der ersten Lektion, die ich vor Jahren gepaukt hatte: «Der Hahn kräht auf dem Misthaufen", "Gallus in sterquilinio cantat». Welcher Idiot liess uns Misthaufen als lateinische Vokabel lernen, ein Wort, das kein Mensch je brauchen würden? Plötzlich wurde mir mit Schrecken klar, dass ich ja gar nicht auf dem Schulweg war. Aber wo war ich dann? Ich versuchte, die Augen zu öffnen: diesmal gelang es mir. Ich lag in einem völlig weissen Zimmer, ohne Bilder an der Wand, in einem Bett mit Bettgittern. Ich versuchte, den Kopf zu heben, um meine Umgebung besser zu sehen. Das Bewegen war aber nicht möglich, denn sofort fuhr ein stechender Schmerz vom Nacken in meinen Hinterkopf und ein Kribbeln in meine Arme. Mein Kopf musste in einer Art Schale liegen. Ich versuchte, die Hände zu heben, da merkte ich, dass sie mit Lederriemen angebunden waren. Die Angst in mir wurde zu Panik. Wo war ich?

Ich hatte Bücher über russische Gefängnisse gelesen, aber ich war noch nie in Russland gewesen. «Wahrscheinlich bin ich im Spital.» Der Gedanke beruhigte mich ein wenig, aber da wird man doch nicht festgebunden. Plötzlich fiel mir ein, dass jemand gesagt hatte, ich oder wer auch immer sollte froh sein, wenn ich sterbe, da ich nie mehr normal sein würde. Dieser Satz traf mich nun wie ein Schlag. War tatsächlich ich damit gemeint? Wer hatte das gesagt? «Nein!», wollte ich rufen, aber es kam nur ein Krächzen aus meinem trockenen, schmerzenden Hals. Ich versuchte, noch einmal den Kopf zu heben, wieder dieser stechende Schmerz, dann wurde es dunkel um mich herum.

\section{Ein freundliches Gesicht}

Etwas oder jemand rieb an meinem Kinn, es roch nach Benzin. Langsam öffnete ich die Augen. «Bist du wach? Ich hatte so gehofft, dass du aufwachst. Muss noch die Klebstoffreste des Sauerstoffschlauchs von deinem Kinn entfernen, bin gleich fertig.» Eine junge Frau beugte sich über mich, ein volles Gesicht mit dunklen Augen, das mich freundlich und etwas überrascht ansah. Die braunen Haare waren straff zu einem Pferdeschwanz gebunden. «Wer bist du?», fragte ich, froh, mit jemandem sprechen zu können. «Ich bin Schwester Cornelia», antwortete sie. Oh, da hätte ich wohl Sie sagen müssen, kam es mir in den Sinn.

Aber Cornelia fuhr gleich weiter: «Du kannst mir ruhig Du sagen, bin ja nicht einmal drei Jahre älter als du. Alle nennen mich hier Conny, ich bin Schwesternschülerin im zweiten Lehrjahr. Wie schön, dass du endlich aus dem Koma erwacht bist.» «Koma?», fragte ich. «Was

\section{Mein Denken funktionierte so langsam,} klebrig, und mein Gehirn war immer noch in der Schule.

ist denn passiert? Wo bin ich?» «Ja, du warst über vier Wochen bewusstlos und wurdest beatmet. Erst vor zwei Tagen konnte ich dich aus der Intensivstation in dieses Zimmer holen. Der Chef meinte, dass du wahrscheinlich gar nicht mehr aus dem Koma erwachen würdest. Deshalb freue ich mich so, dass du nun wach bist.» Da wurde die Tür geöffnet, eine ältere Schwester 


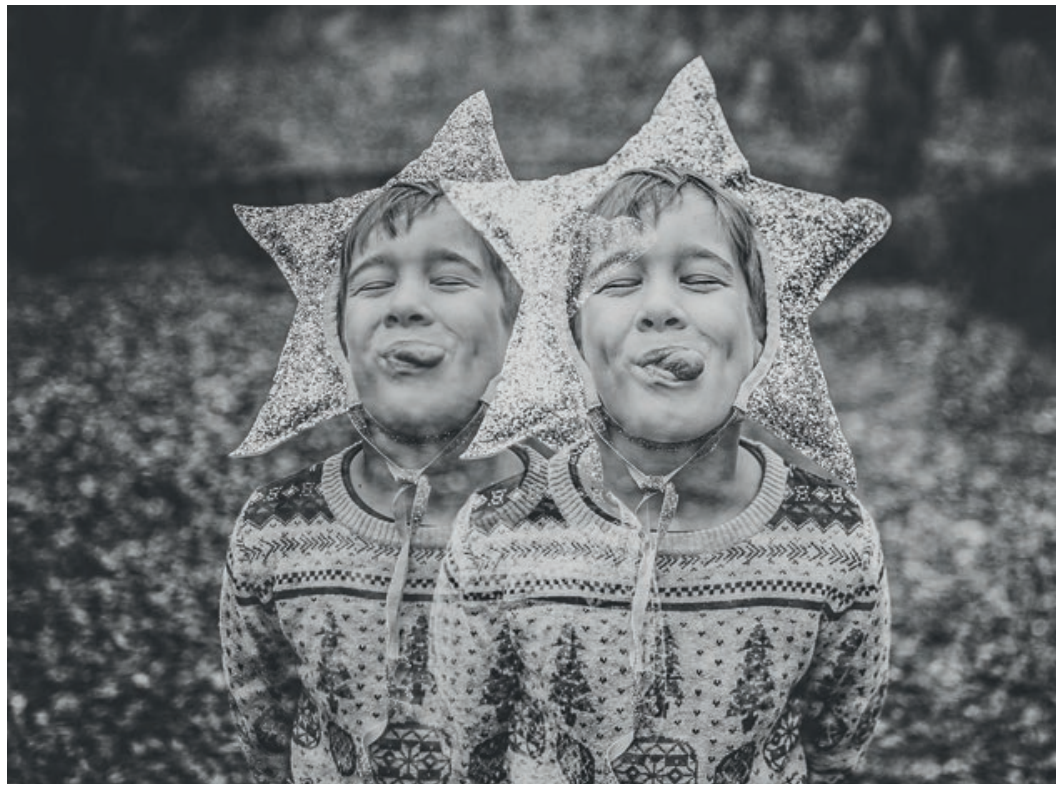

rief: «Hey, Conny, was machst du so lange hier drin, du solltest doch nur schnell eine Zimmerkontrolle machen, wir haben noch viel zu tun.» Conny rief: "Ich komme sofort!» Und zu mir: «Ich habe heute Frühdienst, da kann ich um vier Uhr gehen. Dann komme ich nochmals zu dir! Ich muss auch melden, dass du wach bist.» "Danke», sagte ich. "Melde es bitte nicht, ich brauche noch etwas Zeit, um zu mir zu kommen.» Conny gab keine Antwort, winkte und verschwand durch die Tür.

Vier Wochen im Koma, ich konnte es nicht glauben. Was war geschehen? Ich suchte verzweifelt in meinem Gehirn nach Erinnerungen. Aber ausser der Lateinprüfung heute und noch zwei Prüfungen in Mathe und Physik vor den Herbstferien kam mir nichts in den Sinn. Ach, doch! In den Herbstferien wollte ich mit meiner Schwester eine Wanderung über den Jura nach Biel machen.

Vier Wochen bewusstlos? Da müssten die Herbstferien schon vorbei sein. Mein Denken funktionierte so langsam, klebrig, und mein Gehirn war immer noch in der Schule. Plötzlich kam wieder die Erinnerung an diese seltsame Gruppe um mein Bett herum. War es heute, oder gestern oder vor einigen Tagen gewesen? Was hatte der Mann mit der tiefen Stimme gesagt? Diese Erinnerung kam sofort: Als wäre er noch im Zimmer, hörte ich ihn sagen: «Seien Sie froh, wenn er stirbt. Er wird nie wieder normal sein.»

\section{Zum Sterben noch nicht bereit}

Aber ich wollte normal sein! Ich wollte noch so viel erleben, das Gymnasium beenden, ein Studium machen, vor allem wollte ich nicht sterben! Ich hatte doch noch gar nicht richtig gelebt. Etwas würgte in meinem Hals, der ganz trocken war. Oder ist «nicht normal sein» noch schlimmer als sterben?

In meiner Familie wäre es sicher sehr schwierig, behindert oder schwachsinnig zu sein. Meine Schwestern litten schon unter dem Leistungsdruck von zu Hause, weil sie nicht so begabt waren. Die Gedanken plagten mich. Vielleicht war ich jetzt nicht mehr normal, ich konnte mich an nichts mehr erinnern, lag angebunden in einem Spitalbett. Aber zum Sterben war ich nicht bereit. Nein, ich wollte leben!

Schweiss trat auf meine Stirn, ich konnte ihn nicht einmal abwischen. Wahrscheinlich war es die Angst. Da kam mir in den Sinn, dass Conny gesagt hatte, sie würde nochmals zu mir kommen, wenn ihr Dienst fertig sei. Endlich ein Lichtblick!

Wenn ich mit den Augen ganz nach rechts schaute, denn den Kopf konnte ich nicht drehen, gelang es mir, aus dem Fenster zu blicken. Es war neblig, trotzdem konnte ich wenigstens ein Stück hinauf auf die Jurahöhen sehen, auf denen ich so gerne wandern ging. «Herr, hilf mir», betete ich. Hörte Gott denn zu? Würde ich zu ihm kommen, wenn ich sterben müsste?

\section{Erfahren, was passiert ist}

Ich musste wieder geschlafen haben, denn als ich erwachte, strich mir jemand über die Stirn mit einem nassen Lappen.

Es war Conny. Nun hatte sie die Haare offen, so war sie noch viel hübscher. «Du bist ja ganz verschwitzt», flüsterte sie. «Habe ich dich geweckt? Wie fühlst du dich?» «Ich habe Durst», krächzte ich. Conny gab mir mit einem Schnabelbecher zu trinken. Ihre Nähe tat mir gut. "Conny», wagte ich, sie zu fragen: «Was ist passiert?

\section{Aber ich kam nicht weg von diesen Worten:}

Sterben, nicht mehr normal sein, keine Chance. Sie hatten sich bei mir eingebrannt.

Warum ist es besser zu sterben? Warum werde ich nie mehr normal sein?» «Mein Gott, hast du etwa mitbekommen, was der Professor gesagt hat an der Chefvisite? Warst du da schon bei Bewusstsein?» Sie sah mich erschrocken an. «Dann war das die Chefvisite?» «Der grosse Mann ist der Chef, Prof. L., der kleine, runde ist der Oberarzt.» "Ist der Chef der mit der lauten, tiefen Stimme? Ich war da immer noch halb im Koma, konnte nicht sehen, nur hören.» "Ach so, umso schlimmer. Ja, das war der Chef. Der andere, der ihm geantwortet hatte, eine Rehabilitation sei möglich, das ist Dr. La, er ist Tessiner, wirklich nett, und er kann dir sicher viel mehr erklären als ich. Ich finde es so schlimm, dass du 
das alles gehört hast.» Ich schwieg einen Moment, dann wagte ich zu fragen: «Glaubst du auch, dass ich sterben muss - oder behindert sein werde? Warum hat man mich ans Bett gebunden?»

«Dein Kopf ist in einem Diadem-Gips fixiert, denn du hast die obersten beiden Halswirbel gebrochen. Zum Glück ist der zweite auch gebrochen, da du sonst gestorben wärst, so hat man es mir erklärt. Warum das so ist, musst du Dr. La fragen, Wirbelsäule und Gehirn

Ich wollte noch so viel erleben, das Gymnasium beenden, ein Studium machen, vor allem wollte ich nicht sterben!

sind seine Spezialgebiete. Weil du in den letzten zwei Tagen sehr unruhig warst, musste man dich fixieren. Du könntest durch falsche Bewegungen der Halswirbelsäule eine Querschnittlähmung erleiden. Die Halswirbelsäule müsse man noch operieren, meint der Oberarzt."

Das war schon fast zu viel Information, darüber musste ich noch nachdenken, doch ich fragte weiter: «Und wie ist das alles passiert?» "Ich weiss nur, dass es ein Verkehrsunfall war: Du warst mit dem Mofa unterwegs und bist nach links abgebogen, direkt vor ein Auto, und wurdest mit dem Kopf voran auf das Autodach geschleudert.» "Glaubst du, dass ich wieder gesund werde?», bohrte ich nach. "Ich habe schon viel zu viel gesagt, ich weiss es doch nicht, aber ich werde für dich beten.» Connys Stimme klang gequält.

\section{Heilung ist möglich}

Von meinem sonntagschristlichen Hintergrund her wäre es mir früher peinlich gewesen, wenn jemand gesagt hätte, er wolle für mich beten. Aber jetzt hatte das Versprechen etwas Tröstliches und Beruhigendes. Dennoch fragte ich: «Steht es denn so schlecht um mich?»

Conny schaute mich lange an. «Ich glaube schon, aber du musst glauben, und beten, Heilung ist möglich, das hat doch Dr. La auch gesagt, und bei Gott ist sowieso alles möglich. Ich bete oft für meine Patienten, das hilft. Es hat doch auch bei dir geholfen, denn du bist wieder erwacht aus dem Koma. Zudem hilft es auch mir, all das Schwere, das ich täglich sehe, zu verarbeiten.»

Plötzlich kamen mir die Tränen, ich konnte sie einfach nicht mehr zurückhalten. Irgendwie tat es gut, und als ich, wegen der Tränen doch etwas verlegen, Conny ansah, fragte sie: «Habe ich wieder zu viel gesagt? Das hat mir die Oberschwester schon zum Vorwurf gemacht, dass ich mich zu wenig abgrenze, manchmal zu viel sage, aber das ist manchmal so schwierig!» Nun hatte sie auch feuchte Augen. «Nein, Conny, du hast mir sehr geholfen, ich danke dir!» «Nun muss ich gehen», sagte sie schnell. «Morgen habe ich frei, aber übermorgen bin ich wieder da. Ich werde Pater Martin vom Kloster in Olten bitten, dich $\mathrm{zu}$ besuchen. Es wird dir helfen, wenn du mit ihm sprechen kannst.» "Aber ich bin nicht katholisch", meinte ich. "Das ist ihm egal, er besucht alle Patienten, und das tut ihnen gut. Gute Nacht!» Und schon war Conny zur Tür hinaus.

Ich musste noch während Tagen über ihre Worte und meine Situation nachdenken. Sie hatte mir mit ihrer offenen, herzlichen Begegnung so viel geholfen. Ich wollte kämpfen, ich wollte gesund werden. Ein Teil der Angst, die mir die Äusserung auf der Chefvisite eingeflösst hatte, war zwar noch vorhanden, sie würde mich noch lange begleiten. Aber langsam wich diese Angst einem Vertrauen. Einem Vertrauen, das wuchs dank der vielseitigen Hilfe, die mir zuteilwurde: Die liebevolle Betreuung durch das Pflegepersonal, Dr. Las kompetente Entscheidungen, viele Gesprächen mit Pater Martin, durch den Glauben, der langsam in mir wachsen konnte, und natürlich auch durch Connys ganz spezielle Betreuung und ihren Glauben. So sollte Dr. La Recht bekommen: Ich durfte wieder ganz gesund werden, auch wenn es viele Monate dauerte.

\section{Die eindrücklichste Chefarztvisite}

Ich habe als Unterassistent, als Assistenzarzt und als Oberarzt in meiner neunjährigen Spitalzeit viele Chefvisiten erlebt. Keine ist mir so im Gedächtnis geblieben wie die beschriebene. Sicher sind die Chefvisiten heute ganz anders, ich hoffe es jedenfalls: sensibler, menschlicher. Aber da habe ich nach 31 Jahren in der Praxis den Anschluss verloren.

Authentischer Bericht, erlebt im Oktober 1970 im Kantonsspital Olten, die Namen sind mit den Anfangsbuchstaben anonymisiert.

Bildnachweis

Annie Spratt / Unsplash 